\title{
On asymmetric hyperbolic manifolds
}

\author{
D. D. Long; \& A. W. Reid ${ }^{\dagger}$
}

September 16, 2003

\begin{abstract}
We show that for every $n \geq 2$ there exists closed hyperbolic n-manifolds for which the full group of orientation preserving isometries is trivial.

2000 Mathematics Subject Classification 57S25, 57N16
\end{abstract}

\section{Introduction}

Let $\mathbf{H}^{n}$ denote hyperbolic $n$-space, that is the unique connected simply connected Riemannian manifold of constant curvature -1 . By a hyperbolic $n$-manifold we shall mean a quotient $\mathbf{H}^{n} / \Gamma$ where $\Gamma$ is a torsion-free discrete group of isometries of $\mathbf{H}^{n}$. It is well-known that the group of isometries of a finite volume hyperbolic n-manifold is finite. We shall denote the group of isometries (resp. orientation-preserving isometries) of a hyperbolic manifold $M$ by $\operatorname{Isom}(M)\left(\operatorname{resp}\right.$. $\left.\operatorname{Isom}^{+}(M)\right)$. We define a hyperbolic n-manifold to be asymmetric (resp. +asymmetric) if $\operatorname{Isom}(M)=1$ (resp. $\left.\operatorname{Isom}^{+}(M)=1\right)$. It was shown by Greenberg [6] that every finite group is realized as $\operatorname{Isom}^{+}(M)$ for some hyperbolic surface. Indeed, one can realize any finite group as $\operatorname{Isom}(M)$ for some hyperbolic surface. Kojima [8] showed that a similar result holds in dimension 3. In particular +asymmetric and asymmetric examples exist in low dimensions. However, the methods used in these dimensions tend to rely on special features of low dimensions; for example, Teichmüller theory in dimension 2 or Thurston's Dehn surgery theorem in dimension 3 (see also [5] for a different construction of +asymmetric hyperbolic surfaces using coset diagrams). The main result of this note is the generalization of Greenberg's result to all dimensions.

Theorem 1.1 For every $n \geq 2$ there exists a closed + asymmetric hyperbolic $n$-manifold.

As mentioned, since this is well-known in dimensions 2 and 3 we will only consider manifolds of dimension $\geq 4$. This also avoids some inconveniences in the methods of proof. The method of proof will provide infinitely many examples in each dimension. Passage to asymmetric examples seems much harder (see $\S 4$ for more on this).

\section{Preliminaries}

For convenience we record some standard results about hyperbolic manifolds and the theory of finite groups that will be needed in the proof of Theorem 1.1.

\footnotetext{
${ }^{*}$ This work was partially supported by the N. S. F.

${ }^{\dagger}$ This work was partially supported by the N. S. F. and a grant from the Texas Advanced Research Program.
} 


\section{1}

Recall that the full group of isometries of $\mathbf{H}^{n}$ can be identified with $\mathrm{PO}_{0}\left(f_{n} ; \mathbf{R}\right)$ where $f_{n}$ is the quadratic form $\langle 1,1, \ldots 1,-1\rangle$. The group of orientation-preserving isometries of $\mathbf{H}^{n}$ is a subgroup of index 2 in $\mathrm{PO}_{0}\left(f_{n} ; \mathbf{R}\right)$ consisting of those elements of determinant 1 , denoted by $\mathrm{PSO}_{0}\left(f_{n} ; \mathbf{R}\right)$.

Notation: If $G$ is a group we will denote the commutator subgroup of $G$ by $[G, G]$ and if $H<G$, we will denote the normalizer of $H$ in $G$ by $N_{G}(H)$.

The following lemma is well-known, but we include a sketch of the proof for convenience.

Lemma 2.1 Let $\Gamma$ be a discrete subgroup of $\operatorname{PSO}_{0}\left(f_{n} ; \mathbf{R}\right)$. Then $\operatorname{Isom}\left(\mathbf{H}^{n} / \Gamma\right)$ is isomorphic to $N_{\mathrm{PO}_{0}\left(f_{n} ; \mathbf{R}\right)}(\Gamma) / \Gamma$, and Isom ${ }^{+}\left(\mathbf{H}^{n} / \Gamma\right)$ is isomorphic to $N_{\mathrm{PSO}_{0}\left(f_{n} ; \mathbf{R}\right)}(\Gamma) / \Gamma$

Proof: Given $\eta \in N_{\mathrm{PO}_{0}\left(f_{n} ; \mathbf{R}\right)}(\Gamma)$ this induces an isometry $\bar{\eta}$ of $\mathbf{H}^{n} / \Gamma$. For if $p \in \mathbf{H}^{n}$ then

$$
\eta \Gamma p=\eta \Gamma \eta^{-1} \eta p=\Gamma \eta p
$$

The mapping $\eta \rightarrow \bar{\eta}$ is a homomorphism which induces a homomorphism

$$
f: N_{\mathrm{PO}_{0}\left(f_{n} ; \mathbf{R}\right)}(\Gamma) / \Gamma \rightarrow \operatorname{Isom}\left(\mathbf{H}^{n} / \Gamma\right) .
$$

This is a monomorphism since the kernel of the homomorphism $\eta \rightarrow \bar{\eta}$ is $\Gamma$. That $f$ is onto follows from the fact that any isometry $g$ of $\mathbf{H}^{n} / \Gamma$ lifts to an isometry $\phi_{g}$ of $\mathbf{H}^{n}$ which satisfies $\phi_{g} \Gamma \phi_{g}^{-1}=\Gamma$, that is $\phi_{g} \in N_{\mathrm{PO}_{0}\left(f_{n} ; \mathbf{R}\right)}(\Gamma)$. The second claim also follows on noting that an orientation-preserving isometry of $\mathbf{H}^{n} / \Gamma$ lifts to an orientation-preserving isometry of $\mathbf{H}^{n}$.

An immediate corollary of this is the following.

Corollary 2.2 Let $M=\mathbf{H}^{n} / \Gamma$ be a hyperbolic n-manifold. If $N_{\mathrm{PSO}_{0}\left(f_{n} ; \mathbf{R}\right)}(\Gamma)=\Gamma$, then Isom ${ }^{+}(M)=$ 1.

\section{2}

Here we collect some facts about the structure of subgroups of finite simple groups of Lie type. Useful references are [1] and [2].

Let $G$ be a group, and $B$ and $N$, a pair of subgroups of $G$ which form a $(B, N)$-pair for $G$. $B$ is often called a Borel subgroup of $G$. A standard example is the following.

Example: Let $\mathbf{F}$ be a finite field of characteristic $\neq 2,3$. Then a Borel subgroup $\mathcal{B}$ of $\operatorname{PSL}(n, \mathbf{F})$ is the image in PSL of the group of upper triangular matrices in $\mathrm{SL}(n, \mathbf{F})$. Furthermore any Borel subgroup of $\operatorname{PSL}(n, \mathbf{F})$ is conjugate in $\operatorname{PSL}(n, \mathbf{F})$ to $\mathcal{B}$ (see [1] $\S 16)$.

A class of groups of Lie type that we shall make use of are those arising from orthogonal groups. Let $f$ be an $m$-dimensional quadratic form over the finite field $\mathbf{F}$ of cardinality $q=p^{n}$. To simplify some of the discussion we assume $p$ is odd and $m \geq 5$. In the case when $m$ is also odd, there is a unique orthogonal group $\mathrm{O}(m, q)$ up to isomorphism, and when $m$ is even there are two $\mathrm{O}_{ \pm}(m, q)$ (see [14] p 377 Theorem 5.8). Let $\mathrm{SO}(m, q)$ and $\mathrm{SO}_{ \pm}(m, q)$ denote the special orthogonal groups in these cases.

Let $\Omega(m, q)=[\mathrm{O}(m, q), \mathrm{O}(m, q)]$ when $m$ is odd $\left(\operatorname{resp} . \Omega_{ \pm}(m, q)=\left[\mathrm{O}_{ \pm}(m, q), \mathrm{O}_{ \pm}(m, q)\right]\right.$ when $m$ is even). When $m$ is even $\Omega_{ \pm}(m, q)$ has index 2 in $\operatorname{SO}_{ \pm}(m, q)$ and has a center of order 1 or 
2. Let $\mathrm{P} \Omega_{ \pm}(m, q)$ be the central quotient group. When $m$ is odd, $\Omega(m, q)$ is a simple subgroup of $\mathrm{O}(m, q)$ of index 4 . When $m$ is even, $\Omega(m, q)$ is a subgroup of $\mathrm{O}(m, q)$ of index 4 and the quotient $\mathrm{P} \Omega_{ \pm}(m, q)$ is simple whenever (see [2] or [14] pp 383-384 for more details). We sometimes suppress the subscripts \pm , and also use the notation $\Omega(f ; q)$, or $\Omega(f ; \mathbf{F})$ where $\mathbf{F}$ is a finite field, or simply just $\Omega$ when no confusion will arise.

Remarks: 1. It is known that the groups $\mathrm{SO}(m, q), \mathrm{SO}_{ \pm}(m, q)$ and all finite simple groups of Lie type admit a $(B, N)$-pair (see [2]). In the case of $\mathrm{SO}(m, q), \mathrm{SO}_{ \pm}(m, q)$ the groups $B$ arise from the more classical notion of a Borel subgroup of an algebraic group (see [1]).

2. A fact about Borel subgroups that we shall make use of is that they are all self-normalizing. That is, if $G$ is a group with a $(B, N)$-pair as above, then $N_{G}(B)=B$ (see [1] Theorem 11.16 and [2] $\S 8.3)$.

\section{Proof of Theorem 1.1}

We shall assume throughout that $n \geq 4$ so that the form $f_{n}$ is at least 5 dimensional. This will avoid some minor inconveniences with $n=2$ and 3 in the proof. As already remarked the main theorem is already known in these cases, and indeed infinitely many examples are known.

Theorem 1.1 follows from Theorem 3.1 below on noting that [7] shows the existence of cocompact non-arithmetic groups $\Gamma$ in all dimensions. Also recall that if $\Gamma$ is a finite co-volume non-arithmetic subgroup of $\mathrm{PSO}_{0}\left(f_{n} ; \mathbf{R}\right)$, there is a unique maximal group in the $\mathrm{PO}_{0}\left(f_{n} ; \mathbf{R}\right)$ commensurability class of $\Gamma$ (see [11]). We denote this group by $C(\Gamma)$. By passing to the subgroup of index 2 consisting of orientation-preserving isometries in $C(\Gamma)$, we get a maximal discrete subgroup of $\mathrm{PSO}_{0}\left(f_{n} ; \mathbf{R}\right)$ in the $\mathrm{PSO}_{0}\left(f_{n} ; \mathbf{R}\right)$ commensurability class of $\Gamma$, which contains $\Gamma$.

Theorem 3.1 Let $\Gamma$ be a finite co-volume non-arithmetic subgroup of $\mathrm{PSO}_{0}\left(f_{n} ; \mathbf{R}\right)$ which is maximal in its commensurability class in $\mathrm{PSO}_{0}\left(f_{n} ; \mathbf{R}\right)$. Then $\Gamma$ has a torsion-free group $\Delta$ such that $\operatorname{Isom}^{+}\left(\mathbf{H}^{n} / \Delta\right)=1$.

Before commencing with the proof of Theorem 3.1 we make some further comments. Since $\Gamma$ is a finite co-volume subgroup of $\mathrm{PSO}_{0}\left(f_{n} ; \mathbf{R}\right)$ and $n \geq 4$, Mostow Rigidity implies that we can conjugate $\Gamma$ in $\mathrm{PO}_{0}\left(f_{n} ; \mathbf{R}\right)$ so that $\Gamma$ has entries in a number field, $L$ say (see [13]). We will assume, that $L$ is a minimal such field of definition in the sense of [15]. Note that by definition $L \subset \mathbf{R}$. Furthermore, since $\Gamma$ is finitely generated $\Gamma<\operatorname{PSO}_{0}\left(f_{n} ; R\right)$ where $R$ is a subring of $L$ in which a finite number of prime ideals of $L$ are inverted.

Notation: Let $R \subset L$ be as above and $\wp \subset R$ be a prime ideal. Let $\phi_{\wp}: \operatorname{SL}(n, R) \rightarrow \operatorname{SL}(n, R / \wp)$ denote the reduction homomorphism, $B_{\wp}$ denote the subgroup of $\operatorname{SL}(n, R / \wp)$ consisting of upper triangular matrices of $\mathrm{SL}(n, R / \wp)$ and $\Gamma_{0}(\wp)=\phi_{\wp}^{-1}\left(B_{\wp}\right)$. It will also be a standing assumption that the characteristic of $R / \wp$ is different from 2 in what follows.

Lemma 3.2 In the notation above, there are infinitely many prime ideals $\wp$ for which $\mathrm{P}_{0}(\wp)$ is a torsion free subgroup of finite index in $\operatorname{PSL}(n, R)$.

Proof: That $\Gamma_{0}(\wp)$ has finite index is clear. To see that infinitely many choices of primes $\wp$ give rise to torsion-free $\mathrm{P}_{0}(\wp)$ we argue as follows. There are only finitely many possible orders for elements of finite order in $\mathrm{SL}(n, R)$, and so only finitely many possible prime divisors $q_{1}, \ldots, q_{m}$ of these orders. Let $\alpha \in \mathrm{SL}(n, R)$ be a non-central element of finite order for which $\phi_{\wp}(\alpha) \in B_{\wp}$. It will suffice to consider the case that $\alpha$ has prime order $q$ say, with $q \in\left\{q_{1}, \ldots, q_{m}\right\}$. 
Note that since $L$ is assumed to be real, $L$, and hence $R$, contains no roots of unity $\neq \pm 1$. Since $\alpha$ is not central, it cannot be a diagonal element. The eigenvalues of $\phi_{\wp}(\alpha)$ are $q$-th roots of unity, and these lie on the diagonal of $\phi_{\wp}(\alpha)$. Thus to exclude a non-central element of order $q$ in $B_{\wp}$ we must choose a prime $\wp$ for which the finite field $R / \wp$ contains no primitive $q$-th roots of unity. This is achieved as follows.

Let $M=\prod q_{i}$, and let $\zeta_{M}$ be a primitive $M$-th root of unity. Since $L$ does not contain $\zeta_{M}$, $L\left(\zeta_{M}\right)$ is a proper Galois extension of $L$, say $\left[L\left(\zeta_{M}\right): L\right]=d>1$. Now the Tchebotarev Density theorem implies there are infinitely many $L$-primes $\wp$ which are totally inert in $L\left(\zeta_{M}\right)$. That is, there is a unique prime $P$ in $L\left(\zeta_{M}\right)$ for which $\left[R_{L\left(\zeta_{M}\right)} / P: R_{L} / \wp\right]=d$, where $R_{K}$ denotes the ring of integers of the number field $K$. In particular the finite fields $R_{L} / \wp$ do not contain $\zeta_{M}$. This completes the proof.

As remarked in the Example of $\S 2$, all Borel subgroups of $\operatorname{PSL}(n, \mathbf{F})$ are conjugate. Thus if $\mathbf{F}$ is any of the finite fields $R / \wp$ of Lemma 3.2 , any Borel subgroup of $\operatorname{PSL}(n, \mathbf{F})$ is conjugate in $\operatorname{PSL}(n, \mathbf{F})$ to $\mathrm{P} B_{\wp}$. Hence, in the notation of the proof of Lemma 3.2, for the primes constructed in the proof of Lemma 3.2, the pre-image of any conjugate of $B_{\wp}$ in $\mathrm{SL}(n, \mathbf{F})$ will give rise to a torsion-free subgroup of finite index in $\operatorname{PSL}(n, R)$.

Proof of Theorem 3.1: Let $\pi_{\wp}$ denote the restriction of $\phi_{\wp}$ (as above) to $\operatorname{SO}\left(f_{n} ; R\right)$ and $\hat{\pi}_{\wp}$ denote the induced homomorphism into $\operatorname{PSO}\left(f_{n} ; R / \wp\right)$. Apart from a finite number of primes, this defines homomorphisms $\hat{\pi}_{\wp}: \Gamma \rightarrow \operatorname{PSO}\left(f_{n} ; R / \wp\right)$. The following result is proved in [9]. It is a special case of more general results due to Weisfeiler [16] and Nori [12].

Theorem 3.3 In the notation above, let $q$ denote the cardinality of the residue class field $R_{L} / \wp$. Then for all but a finite number of primes $\wp$, we have $\mathrm{P} \Omega \leq \hat{\pi}_{\wp}(\Gamma) \leq \mathrm{PO}(n+1 ; q)$.

By Theorem 3.3, for all but a finite number of primes $\wp$, the group $\hat{\pi}_{\wp}(\Gamma)$ coincides with exactly one of $\operatorname{PSO}\left(f_{n} ; R / \wp\right)$ or $\mathrm{P} \Omega$. Consider a prime $\wp$ for which $R / \wp$ has odd characteristic and which satisfies the conclusion of Lemma 3.2 (there are infinitely many such primes).

The existence of a Borel subgroup allows us to argue as follows. Let $\mathrm{PB}_{\wp}$ be a Borel subgroup of $\hat{\pi}_{\wp}(\Gamma)$. This is contained in some Borel subgroup of $\operatorname{PSL}(n, R / \wp)$. For any Borel subgroup of $\hat{\pi}_{\wp}(\Gamma)$ is solvable by definition, and a Borel subgroup of $\operatorname{PSL}(n, R / \wp)$ is a maximal solvable subgroup (see [1] Chapter 11). It therefore follows from Lemma 3.2 and the discussion following it, that the pre-image of $\mathrm{PB}_{\wp}$ in $\Gamma$ will be torsion-free. Denote this subgroup by $\Delta_{0}(\wp)$. Furthermore, by Remark 2 of $\S 2$, the subgroup $\mathrm{PB}_{\wp}$ is a self-normalizing subgroup of $\hat{\pi}_{\wp}(\Gamma)$. It follows that $N_{\Gamma}\left(\Delta_{0}(\wp)\right)=\Delta_{0}(\wp)$. Indeed, since $\Gamma$ is non-arithmetic, $\Delta_{0}(\wp)$ is non-arithmetic and so $N_{\operatorname{PSO}_{0}\left(f_{n} ; \mathbf{R}\right)}\left(\Delta_{0}(\wp)\right) \subset \Gamma$. By the usual correspondence theorem for groups and quotient groups, we deduce that;

$$
N_{\mathrm{PSO}_{0}\left(f_{n} ; \mathbf{R}\right)}\left(\Delta_{0}(\wp)\right)=N_{\Gamma}\left(\Delta_{0}(\wp)\right)=\Delta_{0}(\wp) .
$$

Corollary 2.2 completes the proof.

Note that the proof of Theorem 3.1 makes clear that there are infinitely many such subgroups $\Delta$.

\section{Final Remarks}

1. The method above does not work for arithmetic groups. For example even in the case of $\operatorname{PSL}(2, \mathbf{Z})$ and $\wp=p$ is a rational prime, the normalizer of the groups $\operatorname{P}_{0}(p)$ in $\operatorname{PSL}(2, \mathbf{R})$ properly contains $\mathrm{P}_{0}(p)$. These groups are maximal subgroups in the commensurability class of $\operatorname{PSL}(2, \mathbf{Z})$. 
2. Arithmetic constructions can be used to provide asymmetric examples in low dimensions. For example the work of Chinburg and Friedman [3] constructs maximal arithmetic Kleinian groups which are torsion free. We do not know whether such examples can be built in higher dimensions.

3. The passage to asymmetric examples seems harder. To make the above proof work, one needs to construct a non-arithmetic group $\Gamma$ for which the group $C(\Gamma)$ is a $\operatorname{subgroup~of~} \operatorname{PSO}\left(f_{n} ; \mathbf{R}\right)$. However, as far as we are aware, the constructions of non-arithmetic lattices known do not afford the control needed to arrange this. From this we leave as a question:

Question: For every $n \geq 4$ does there exist an example of a finite volume non-arithmetic hyperbolic n-manifold that has no non-orientable quotients?

4. The more general question of whether every finite group acts as the full group of isometries of a closed hyperbolic $n$-manifold also seems harder in dimensions $\geq 4$. One can use surjections (at least virtually) from the non-arithmetic groups of [7] onto free groups (see [10]) to easily arrange that every finite group is a subgroup of the full group of isometries. At present we do not know how to control this construction so as to obtain the finite group as the full group of isometries.

5. Finally we comment on the situation for other rank 1 symmetric spaces. The proof of Theorem 3.1 can be modified to exhibt +asymmetric complex hyperbolic manifolds (where + denotes the holomorphic isometries in this case), so long as non-arithmetic manifolds exist. In particular, given the existence, one can modify Lemmas 3.2 and apply the results of [12] and [16] in the context of the finite simple groups of PSU-type to generalize Theorem 3.3. At present non-arithmetic complex hyperbolic manifolds are known to exist of dimension 2 and 3 (see [4]). For the case of quaternionic hyperbolic $n$-space $(n \geq 2)$ and the Cayley hyperbolic plane, all finite co-volume groups are arithmetic and so our methods do not provide closed +asymmetric manifolds for these geometries.

Furthermore, since finite co-volume groups acting on quaternionic hyperbolic n-space $(n \geq 2)$ and the Cayley hyperbolic plane have Property T, there are no finite co-volume groups admitting homomorphisms onto non-trivial free groups. Furthermore, if the Congruence Subgroup Property holds for these groups (this is at present still an open problem), then there will be finite groups that cannot appear even as subgroups of the full group of isometries of such manifolds.

\section{References}

[1] A. Borel, Linear Algebraic Groups, Graduate Texts in Math. 126 Springer, (1991).

[2] R. Carter, Simple Groups of Lie Type, John Wiley and Sons (1989).

[3] T. Chinburg and E. Friedman, The finite subgroups of maximal arithmetic subgroups of PGL(2, C), Ann. Inst. Fourier 50 (2000), pp. 1765-1798.

[4] P. Deligne and G. D. Mostow, Commensurabilities among lattices in $\mathrm{PU}(n, 1)$, Annals of Math. Studies 132 Princeton University Press (1993). 
[5] B. Everitt, A family of conformally asymmetric Riemann surfaces, Glasgow Math. J. 39 (1997), pp. 221-225.

[6] L. Greenberg, Maximal groups and signatures, Annals of Math. Studies 79 Princeton University Press (1993), pp. 207-226.

[7] M. Gromov and I. Piatetski-Shapiro, Nonarithmetic groups in Lobachevsky spaces, Publ. I.H.E.S. 66 (1988), pp. 93-103.

[8] S. Kojima, Isometry transformations of hyperbolic 3-manifolds, Topology and its Appl. 29 (1988), pp. 297-307.

[9] D. D. Long and A. W. Reid, Constructing hyperbolic manifolds which bound geometrically, Math. Research Letters 8 (2001), pp. 443-455.

[10] A. Lubotzky, Free quotients and the first betti number of some hyperbolic manifolds, Transformation Groups 1 (1996), pp. 71-82.

[11] G. Margulis, Discrete Subgroups of Semi-simple Lie Groups, Ergeb. der Math. 17 SpringerVerlag (1989).

[12] M. V. Nori, On subgroups of $\mathrm{GL}_{n}\left(\mathbf{F}_{p}\right)$, Invent. Math. 88 (1987), pp. 257-276.

[13] M. S. Raghunathan, Discrete Subgroups of Lie Groups, Ergebnisse der Mathematik und ihrer Grenzgebiete, 68 Springer-Verlag (1972).

[14] M. Suzuki, Group Theory I, Grundlehren der mathematischen Wissen. 247, Springer-Verlag, (1982).

[15] E. B. Vinberg, Rings of definition of dense subgroups of semisimple linear groups, Math. USSR Izvest. 5 (1972), pp. 45-55.

[16] B. Weisfeiler, Strong approximation for Zariski dense subgroups of semi-simple algebraic groups, Annals of Math. 120 (1984), pp. 271 - 315.

Department of Mathematics,

University of California

Santa Barbara, CA 93106

Department of Mathematics,

University of Texas

Austin, TX 78712 